\title{
High throughput whole mitochondrial genome sequencing by two platforms of massively parallel sequencing
}

\author{
Seung Bum Seo ${ }^{1}$, Xiangpei Zeng ${ }^{1}$, Mourad Assidi ${ }^{2,3}$, Bobby LaRue ${ }^{1}$, Jonathan King ${ }^{1}$, Antti Sajantila ${ }^{1,4}$, \\ Bruce Budowle $e^{1,2^{*}}$
}

From 2nd International Genomic Medical Conference (IGMC 2013)

Jeddah, Kingdom of Saudi Arabia. 24-27 November 2013

\section{Background}

Mitochondrial DNA (mtDNA) is a valuable genetic biomarker that has been implicated as a prognostic/diagnostic indicator for a number of diseases [1-3] as well as for human identification where forensic biologic evidence contains too little or no nuclear DNA, such as a hair shaft without root or a fingernail, or where a sample from a distant maternal relative is the only possibility for comparison [4-6]. Sanger sequencing has been the gold standard method for mtDNA typing, but the methodology has limitations with throughput, scalability, speed, and resolution [7]. Massively parallel sequencing technology (MPS) provides platforms for more comprehensive coverage of the genome per sample analyzed than currently is possible with Sanger sequencing [8,9]. Moreover, a number of different samples, which can be distinguished by barcoding, may be sequenced simultaneously. Two of the available personal genome sequencers are the Ion Torrent Personal Genome Machine (PGM ${ }^{\mathrm{TM}}$ ) (LifeTechnologies, San Francisco, CA) and the MiSeq ${ }^{\mathrm{TM}}$ (Illumina, Inc., San Diego, CA). The PGM exploits non-optical sequencing on CMOS integrated circuits by detecting small changes in $\mathrm{pH}$, due to release of $\mathrm{H}^{+}$during addition of a nucleotide to the growing strand within a 2 hour run time. The MiSeq uses fluorescently tagged terminator chemistry and requires 39 hours for paired-end sequencing but has higher throughput and an associated simpler, less labor intensive library preparation methodology than the PGM.

\footnotetext{
* Correspondence: bruce.budowle@unthsc.edu

'Institute of Applied Genetics, Department of Forensic and Investigative Genetics, University of North Texas Health Science Centre, 3500 Camp Bowie Blvd., FoCentreth, Texas 76107, USA

Full list of author information is available at the end of the article
}

With MPS potentially the mitochondrial genomes of 96 samples could be sequenced at one time. Sequencing of the entire mitochondrial genome provides higher resolution and discrimination power than is currently possible with only sequencing portions of the non-coding region of the mitochondrial genome (for high information content) or by targeted analyses (for a few SNP or deletions noted in the coding region).

\section{Materials and methods}

DNA was extracted (QIAamp DNA Blood Mini Kit, Qiagen, Hilden, Germany) from whole blood of volunteers with informed consent. Whole genome mtDNA was amplified using primers that generate two amplicons approximately $\sim 8.5 \mathrm{~kb}$ in length [9]. The general workflow is displayed in Figure 1.

The protocol for whole mitochondrial genome sequencing on the PGM is described on ion community (http://ioncommunity.lifetechnologies.com/community/ applications/hid/mito/how_to). Ion Xpress ${ }^{\mathrm{TM}}$ Plus gDNA Fragment Library Kit, OneTouch ${ }^{\mathrm{TM}} 200$ Template Kit v2 and Ion PGM ${ }^{\mathrm{TM}} 200$ Sequencing Kit were mainly used for the library preparation, template preparation and sequencing reactions, respectively. On the MiSeq, the amplified DNA was fragmented and tagged using the NexteraXT protocol, indexed, size selected, and pooled for sequencing using the small amplicon targeted resequencing run, which performs $251 \mathrm{bp}$ paired end sequencing reads, according to the manufacturer's recommendations

(http://supportres.illumina.com/documents/myillumina/ 900851dc-01cf-4b70-9e95-d590531c5bd4/nextera_xt_sample_preparation_guide_15031942_c.pdf); (http://support. 


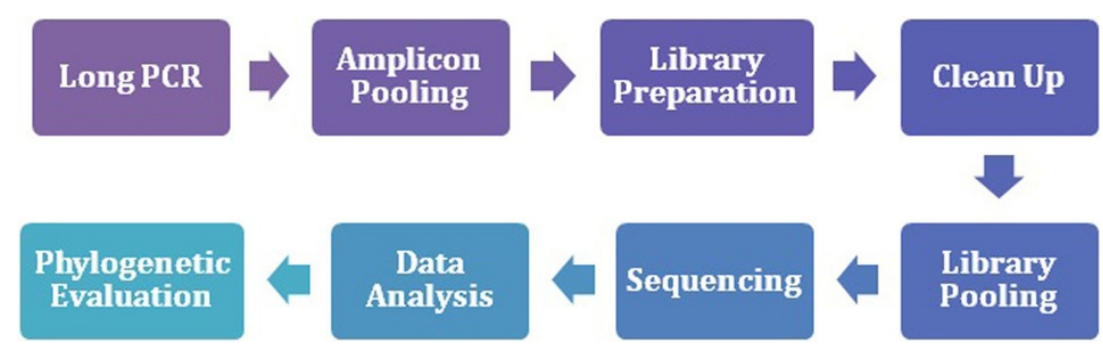

Figure 1 Basic MPS workflow for mtDNA sequencing.

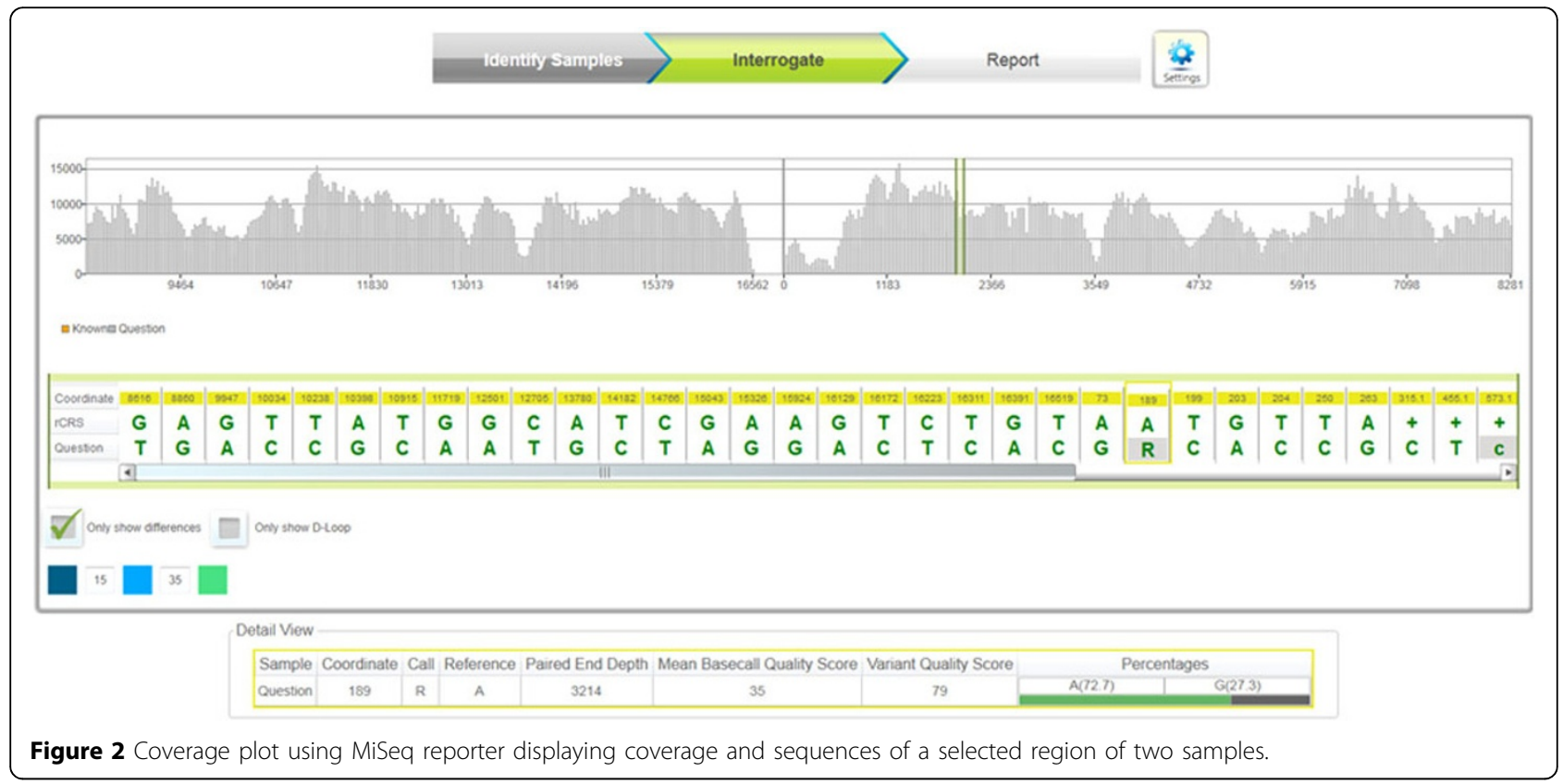

illumina.com/sequencing/sequencing_instruments/miseq/ training.ilmn) analyzed with the PGM platform and Ion $314^{\mathrm{TM}}$ Chip and 48 samples were analyzed on the MiSeq. Therefore, 23 samples were in common between the two MPS systems. In this study, sequencing of DNA was assessed for throughput, coverage, concordance of results between platforms, resolution of heteroplasmy and interpretation of homopolymeric stretch regions.

\section{Results}

Average coverage of the 23 samples was at least 490X in the reactions (Figure 2):

Most variants were concordant between two different MPS platforms and with non-coding region data generated by Sanger sequencing. High quality phylogenetic scores $(>89 \%)$ were obtained for the typing results from all samples with Haplogrep [10] (http://haplogrep.uibk. ac.at). While final calls were corrected manually, some regions were problematic. Most of these problematic areas were located at homopolymer regions due to base position shift and heteroplasmy. In addition, read strand bias was observed at several locations. Many of the discordant results can be corrected by software improvements.

\section{Conclusions}

Overall, the PGM and MiSeq approaches generated good quality sequence data rapidly with relatively high coverage. The progress on these studies will be presented to provide insight on the near term applications and long term potential utility of MPS for both prognostic and diagnostic applications.

\section{Authors' details}

'Institute of Applied Genetics, Department of Forensic and Investigative Genetics, University of North Texas Health Science Centre, 3500 Camp Bowie Blvd., FoCentreth, Texas 76107, USA. ${ }^{2}$ Center of Excellence in Genomic Medicine Research, King Abdulaziz University, Jeddah, Saudi Arabia. ${ }^{3}$ KACST Technology Innovation Centre for Personalized Medicine at King AbdulAziz University, Jeddah, Saudi Arabia. ${ }^{4}$ Department of Forensic Medicine, Hjelt Institute, University of Helsinki, Helsinki, Finland. 


\section{References}

1. El-Hattab AW, Scaglia F: Mitochondrial DNA depletion syndromes: review and updates of genetic basis, manifestations, and therapeutic options. Neurotherapeutics. Journal of the American Society for Experimental NeuroTherapeutics 2013, 10(2):186-198.

2. Silkjaer T, Norgaard JM, Aggerholm A, Ebbesen LH, Kjeldsen E, Hokland P, Nyvold CG: Characterization and prognostic significance of mitochondrial DNA variations in acute myeloid leukemia. European journal of haematology 2013, 90(5):385-396.

3. Uzawa K, Baba T, Uchida F, Yamatoji M, Kasamatsu A, Sakamoto Y, Ogawara K, Shiiba M, Bukawa H, Tanzawa H: Circulating tumor-derived mutant mitochondrial DNA: a predictive biomarker of clinical prognosis in human squamous cell carcinoma. Oncotarget 2012, 3(7):670-677.

4. Ginther $C$, Issel-Tarver $L$, King MC: Identifying individuals by sequencing mitochondrial DNA from teeth. Nature Genetics 1992, 2(2):135-138.

5. Wilson MR, Polanskey D, Butler J, DiZinno JA, Replogle J, Budowle B: Extraction, PCR amplification, and sequencing of mitochondrial DNA from human hair shafts. BioTechniques 1995, 18:662-669.

6. Nunez C, Baeta M, Sosa C, Casalod Y, Ge J, Budowle B, Martinez-Jarreta B: Reconstructing the population history of Nicaragua by means of mtDNA, Y-chromosome STRs, and autosomal STR markers. American journal of physical anthropology 2010, 143(4):591-600.

7. Sanger F, Nicklen S, Coulson AR: DNA sequencing with chain-terminating inhibitors. Proceedings of the National Academy of Sciences USA 1997, 74(12):5463-5467.

8. Berglund EC, Kiialainen A, Syvänen AC: Next-generation sequencing technologies and applications for human genetic history and forensics. Investigative Genetics 2011, 24:2-23.

9. Gunnarsdóttir ED, Li M, Bauchet M, Finstermeier K, Stoneking M: Highthroughput sequencing of complete human mtDNA genomes from the Philippines. Genome Research 2011, 21(1):1-11.

10. Kloss-Brandstätter A, Pacher D, Schönherr S, Weissensteiner H, Binna R, Specht G, Kronenberg F: HaploGrep: A fast and reliable algorithm for automatic classification of mitochondrial DNA haplogroups. Human Mutation 2011, 32(1):25-32.

doi:10.1186/1471-2164-15-S2-P7

Cite this article as: Seo et al.: High throughput whole mitochondrial genome sequencing by two platforms of massively parallel sequencing. BMC Genomics 2014 15(Suppl 2):P7.

\section{Submit your next manuscript to BioMed Central and take full advantage of:}

- Convenient online submission

- Thorough peer review

- No space constraints or color figure charges

- Immediate publication on acceptance

- Inclusion in PubMed, CAS, Scopus and Google Scholar

- Research which is freely available for redistribution

Submit your manuscript at www.biomedcentral.com/submit
Biomed Central 